\title{
Predictors of excellent functional outcome in aneurysmal subarachnoid hemorrhage
}

\author{
Marianna Pegoli, MD, ${ }^{1}$ Jay Mandrekar, PhD, ${ }^{2}$ Alejandro A. Rabinstein, MD, ${ }^{3}$ \\ and Giuseppe Lanzino, MD' \\ 'Departments of Neurosurgery, ${ }^{2}$ Biomedical Statistics and Informatics, and ${ }^{3}$ Neurology, Mayo Clinic, Rochester, Minnesota
}

\begin{abstract}
OBJECT Case fatality rates after aneurysmal subarachnoid hemorrhage (aSAH) have decreased over time, and many patients treated with modern paradigms return to a normal life. However, there is little information on predictors of excellent functional outcome after aSAH. In this study, the authors investigated predictors of excellent outcome in a modern consecutive series of patients with aSAH.
\end{abstract}

METHODS A retrospective review was conducted of patients with aSAH admitted between 2001 and 2013. The primary outcome measure was excellent functional outcome, defined as modified Rankin Scale (mRS) score of 0 or 1 at last follow-up within 1 year of aSAH.

RESULTS Three hundred seventy-three patients were identified with posthospital follow-up. Excellent outcome was noted in 236 patients (63.3\%), including an mRS score of 0 in 122 (32.7\%) and an mRS score of 1 in 114 (30.6\%). On univariate analysis, the following factors were associated with an excellent outcome: indicators of less severe bleeding, such as better World Federation of Neurosurgical Societies grade at any of the times of assessment, better modified Fisher grade, and absence of intracerebral hemorrhage (ICH), intraventricular hemorrhage (IVH), and symptomatic hydrocephalus; aneurysm treatment with coil embolization; absence of symptomatic vasospasm, delayed cerebral ischemia, and radiological infarction; absence of in-hospital seizures; lack of need for CSF diversion; fewer hours with fever; less severe anemia; and absence of transfusion. On multivariable analysis, the 4 variables that were most strongly associated with excellent outcome were presence of good clinical grade after neurological resuscitation, absence of $\mathrm{ICH}$ on initial CT scan, blood transfusion during the hospitalization, and radiological infarctions on final brain imaging.

CONCLUSIONS Excellent outcomes (mRS score 0-1) can be achieved in the majority of patients with aSAH. The likelihood of excellent outcome is predicted by good clinical condition after resuscitation, absence of ICH on presentation, no evidence of infarction on brain imaging, and absence of blood transfusion during hospitalization.

http://thejns.org/doi/abs/10.3171/2014.10.JNS14290

KEY WORDS subarachnoid hemorrhage; outcome; prediction; prognosis; vascular disorders

A LTHOUGH the incidence of aneurysmal subarachnoid hemorrhage (aSAH) has remained stable over time, case fatality rates have decreased by $17 \%$ during the last three decades..$^{11,18}$ In parallel with refinements in endovascular and surgical treatment and advances in neurocritical care, there has also been an improvement in functional outcome..$^{2,16,19,23}$ There are many studies on factors predictive of poor functional and cognitive outcome after aSAH, $, 5,6,22,25$ but much less information on predictors of excellent outcome. In this study, we analyzed predictors of excellent functional outcome in a contemporary series of patients with aSAH.

\section{Methods Study Population}

After Institutional Review Board approval from the Mayo Clinic, we performed a retrospective chart review of adult patients with aSAH admitted to St. Mary's HospitalMayo Medical Center in Rochester, Minnesota, between February 2001 and June 2013. Subarachnoid hemorrhage (SAH) was confirmed radiologically or by the presence of xanthochromia in the CSF. Only patients with a documented aneurysm on cerebral angiography that was considered to be the cause of the SAH were included.

ABBREVIATIONS aSAH = aneurysmal SAH; DCI = delayed cerebral ischemia; ICH = intracerebral hemorrhage; IVH = intraventricular hemorrhage; $\mathrm{mRS}=$ modified Rankin Scale; SAH = subarachnoid hemorrhage; WFNS = World Federation of Neurosurgical Societies.

SUBMITTED February 11, 2014. ACCEPTED October 22, 2014.

INCLUDE WHEN CITING Published online December 12, 2014; DOI: 10.3171/2014.10.JNS14290.

DISCLOSURE Dr. Lanzino has served as a consultant to ev3/Covidien, Edge Therapeutics, and Codman. 


\section{Outcome Measures}

Clinical severity was classified using the World Federation of Neurosurgical Societies (WFNS) grade assigned at presentation, at the time of worst clinical condition, and after neurological resuscitation. Extension of blood on CT was graded according to the modified Fisher scale.

Our multidisciplinary management of aSAH has been described elsewhere. ${ }^{21}$ The type of aneurysm treatment (microsurgical clip placement versus endovascular occlusion of the aneurysm) was decided by consensus of a multidisciplinary team based on aneurysm characteristics and neurological and systemic status of the patient. Surveillance for vasospasm was conducted using transcranial Doppler ultrasonography (in which mean arterial velocities exceeding $120 \mathrm{~cm} / \mathrm{sec}$ in the intracranial internal carotid, anterior cerebral, or middle cerebral arteries were considered suggestive of vasospasm), CT angiography, or catheter angiography (unequivocal reduction in luminal diameter; severe when narrowing was $>50 \%$ ). In cases of symptomatic vasospasm, patients were treated with hemodynamic augmentation. Refractory cases were treated with intraarterial vasodilators or mechanical angioplasty.

Delayed cerebral ischemia (DCI) was defined by the presence of otherwise unexplained clinical deterioration or a new infarct on brain imaging that was not visible on the admission or on the early postoperative scans. Other potential causes of clinical deterioration were rigorously excluded, such as hydrocephalus, rebleeding, or seizures. Every patient with clinical deterioration underwent at least one form of brain imaging (CT or MRI) after symptom onset to evaluate for structural changes.

Functional outcome was assessed using the modified Rankin Scale (mRS) score based on information abstracted by 1 of the investigators (M.P.) who was not involved in the treatment of any of the patients. The primary outcome measure of our analysis was excellent functional outcome, defined as mRS score of 0 or $1(0=$ no symptoms at all, $1=$ no significant disability despite symptoms and able to carry out all usual duties and activities) at last follow-up within 1 year of the aSAH.

The analysis included investigation of age, smoking status, medical comorbidities, Glasgow Coma Scale score, WFNS, modified Fisher grade, aneurysm localization, intraventricular hemorrhage (IVH), intracerebral hemorrhage (ICH), aneurysm treatment, neurological and systemic complications, radiological infarction, and transfusion. Neurological complications included rebleeding, symptomatic vasospasm, DCI, seizures, and hydrocephalus. Systemic complications included fever load, defined as the number of hours with temperature greater than $38^{\circ}$ while in the intensive care unit; anemia, defined as hemoglobin les than $8 \mathrm{~g} / \mathrm{dl}$; and acute lung injury/acute respiratory distress syndrome, defined by a $\mathrm{P} / \mathrm{F}$ ratio $>150$, or 200 with bilateral noncardiogenic lung infiltrates.

\section{Statistical Analysis}

Descriptive summaries were reported as mean \pm standard deviation for continuous variables and as frequency (percentage) for categorical variables. Associations between all the variables mentioned in the previous paragraph and $\mathrm{mRS}$ score of 0 to 1 (yes/no) as a binary out- come were assessed using univariate logistic regression analysis. Neither imputation nor extrapolation was used to account for missing variables; if a variable was missing, it was not included in the analysis. Any variable associated with functional outcome with a $\mathrm{p}$ value $\leq 0.10$ on univariate analysis was entered in a stepwise model using multivariable logistic regression analysis. Area under the receiver operating characteristic curve was reported as a measure of model's prediction ability. An area under the receiver operating characteristic curve estimate of $0.7-0.8$ was regarded as acceptable, $0.8-0.9$ was regarded as excellent, and more than 0.9 was regarded as outstanding.

\section{Results}

We identified 381 patients during the study period. The mean age of the patients was $55.8 \pm 13.3$ years and 244 (64\%) were women. Risk factors included hypertension in $212(55.6 \%)$ and active smoking in 198 (52.0\%). The mean WFNS grade at presentation was $2.3 \pm 1.5$ and after neurological resuscitation was $2.1 \pm 1.5$. The mean modified Fisher grade was $3.0 \pm 0.9$, with ICH in 81 cases (21.3\%) and IVH in 190 (49.9\%). Cerebrospinal fluid diversion for hydrocephalus was necessary in 226 patients (59.3\%). Coil embolization was the aneurysm treatment method in 245 cases $(64.3 \%)$. DCI was diagnosed in 136 patients $(35.7 \%)$ and radiological infarctions were documented in $116(30.4 \%)$.

There were 373 patients with posthospital followup evaluation. The mean last follow-up within 1 year of aSAH was $5.5 \pm 3.8$ months (range 6 weeks to 12 months). Excellent outcome was noted in 236 patients $(63.3 \%)$, including an mRS score of 0 in $122(32.7 \%)$ and an mRS score of 1 in 114 (30.6\%). Among the 216 patients who maintained a good clinical grade (WFNS Grade I-III) at all times between presentation and aneurysm treatment, $174(80.6 \%)$ achieved excellent outcome. Among 105 patients with poor grade at presentation (WFNS Grade IV or V), 33 (31.4\%) had excellent outcomes: 19 (39.6\%) of 48 with Grade IV and 14 (24.6\%) of 57 with Grade V. Among 86 patients with poor grade after neurological resuscitation, $11(12.8 \%)$ had excellent outcomes: $8(19.5 \%)$ of 41 with Grade IV and 3 (6.7\%) of 45 with Grade V. Among 281 patients with modified Fisher grade Grades 3-4, 167 patients $(59.4 \%$ ) had an excellent functional outcome.

Results of the univariate analyses are shown in Table 1. The following variables were associated with better chances of having an excellent outcome: indicators of less severe bleeding (better WFNS grade at any of the times of assessment, better modified Fisher grade, absence of ICH, IVH, or symptomatic hydrocephalus); aneurysm treatment with coil embolization; absence of symptomatic vasospasm, DCI, and radiological infarction; absence of in-hospital seizures; lack of need for CSF diversion; fewer hours with fever; less severe anemia; and absence of transfusion. On multivariable analysis, the 4 variables that were most strongly associated with excellent outcome were the presence of good clinical grade after neurological resuscitation, the absence of ICH on initial CT scan, blood transfusion during hospitalization, and radiological infarctions on final brain imaging (Table 2). The model constituted by 
TABLE 1. Univariate analyses showing association of baseline and hospital variables with excellent favorable outcome (mRS score $0-1)^{*}$

\begin{tabular}{|c|c|c|c|c|}
\hline Variable & $\begin{array}{l}\text { Outcome Other } \\
\text { Than Excellent }\end{array}$ & $\begin{array}{l}\text { Excellent } \\
\text { Outcome }\end{array}$ & OR $(95 \% \mathrm{Cl})$ & $\mathrm{p}$ Value \\
\hline Mean age $\pm S D$ (yrs) & $57.4 \pm 13.7$ & $54.6 \pm 12.7$ & $0.98(0.97-1.0)$ & 0.05 \\
\hline Male sex & $44(32.1)$ & $89(37.7)$ & $1.28(0.82-2.0)$ & 0.28 \\
\hline Hypertension history & $80(58.4)$ & $127(53.8)$ & $0.83(0.54-1.27)$ & 0.39 \\
\hline Active smoking & $65(47.8)$ & $127(53.8)$ & $1.27(0.83-1.94)$ & 0.26 \\
\hline \multicolumn{5}{|l|}{ Mean WFNS grade \pm SD } \\
\hline At presentation & $3.2 \pm 1.6$ & $1.8 \pm 1.2$ & $0.53(0.46-0.62)$ & $<0.01$ \\
\hline At nadir & $3.6 \pm 1.4$ & $2.4 \pm 1.3$ & $0.51(0.43-0.61)$ & $<0.01$ \\
\hline After resuscitation & $3.3 \pm 1.6$ & $1.5 \pm 0.8$ & $0.36(0.29-0.44)$ & $<0.01$ \\
\hline WFNS Grade I-III after neurological resuscitation & $62(45.3)$ & $224(95.3)$ & $24.6(12.3-49.2)$ & $<0.01$ \\
\hline Mean modified Fisher grade \pm SD & $3.4 \pm 0.8$ & $2.8 \pm 1.0$ & $0.49(0.38-0.65)$ & $<0.01$ \\
\hline $\mathrm{ICH}$ & $50(38.5)$ & $30(13)$ & $0.24(0.14-0.40)$ & $<0.01$ \\
\hline IVH & $86(65.6)$ & $102(44.3)$ & $0.42(0.27-0.65)$ & $<0.01$ \\
\hline Symptomatic hydrocephalus & $101(75.4)$ & $129(55.6)$ & $0.41(0.27-0.66)$ & $<0.01$ \\
\hline CSF diversion & $98(72.6)$ & $124(52.5)$ & $0.42(0.27-0.66)$ & $<0.01$ \\
\hline Posterior circulation aneurysm & $29(21.3)$ & $41(17.4)$ & $0.78(0.46-1.32)$ & 0.35 \\
\hline Coil embolization & $76(58.5)$ & $167(71.7)$ & $1.80(1.15-2.82)$ & 0.01 \\
\hline Seizure in hospital & $34(24.8)$ & $23(9.7)$ & $0.33(0.18-0.58)$ & $<0.01$ \\
\hline Symptomatic vasospasm & $44(38.9)$ & $55(23.9)$ & $0.49(0.30-0.80)$ & $<0.01$ \\
\hline $\mathrm{DCl}$ & $64(52.5)$ & $68(29.2)$ & $0.37(0.24-0.59)$ & $<0.01$ \\
\hline Radiological infarction & $65(52.8)$ & $48(20.6)$ & $0.23(0.14-0.37)$ & $<0.01$ \\
\hline Acute lung injury/acute respiratory distress syndrome $†$ & $33(24.1)$ & $37(15.7)$ & $0.59(0.35-0.99)$ & 0.05 \\
\hline Mean hemoglobin level $(\mathrm{g} / \mathrm{dl})$ at nadir $\pm \mathrm{SD}$ & $10.4 \pm 2.2$ & $11.1 \pm 1.7$ & $1.22(1.09-1.37)$ & $<0.01$ \\
\hline Transfusion & $56(40.9)$ & $33(14.0)$ & $0.24(0.14-0.39)$ & $<0.01$ \\
\hline Mean fever load \pm SD $\ddagger$ & $47.0 \pm 70.8$ & $23.8 \pm 45.9$ & $0.99(0.99-1.0)$ & $<0.01$ \\
\hline
\end{tabular}

these 4 variables predicted excellent functional outcome with an area under the curve of 0.85 .

\section{Discussion}

Unlike most previous research on prognosis in aSAH, our study focuses on the prediction of excellent functional outcome. We found excellent functional outcome is very likely in patients who are in good clinical condition after neurological resuscitation, do not have an intraparenchymal hematoma on the first CT scan, do not develop brain infarctions from delayed ischemia, and do not receive a

TABLE 2. Multivariable analyses showing independent associations with excellent favorable outcome (mRS score $0-1$ )

\begin{tabular}{lcc}
\hline \multicolumn{1}{c}{ Variable } & OR $(95 \% \mathrm{Cl})$ & $\mathrm{p} \mathrm{Value}$ \\
\hline $\begin{array}{l}\text { WFNS Grade I-III after neurological } \\
\text { resuscitation }\end{array}$ & $15.94(7.17-5.44)$ & $<0.0001$ \\
\hline $\begin{array}{l}\text { Radiological infarction on final brain } \\
\text { imaging }\end{array}$ & $0.23(0.12-0.41)$ & $<0.0001$ \\
\hline Blood transfusion during hospitalization & $0.31(0.16-0.59)$ & 0.0004 \\
\hline Absence of ICH on initial CT scan & $0.36(0.18-0.70)$ & 0.0028 \\
\hline
\end{tabular}

blood transfusion during the hospital stay. Perhaps most importantly, our results indicate that an excellent functional outcome can be achieved in a majority of patients with aSAH.

Available literature typically addresses the recognition of markers of poor prognosis. ${ }^{5,6,22,25}$ Historically, aSAH has been considered a devastating disease. ${ }^{8,9}$ Surviving it with some function was already considered a success. Yet, advances in treatment now allow us to help patients recover full function in many instances. For that to happen, the therapeutic attitude needs to change from one of satisfaction despite moderate deficits to one that aims at avoiding and correcting all deficits, and regards every patient presenting with good clinical grade and no established brain damage on imaging as someone with the potential to regain a fully functional and productive life.

Few previous studies have reported functional outcomes with sufficient detail to compare with our results. In the multicenter International Study of Aneurysm Treatment (ISAT), $49 \%$ of patients has an mRS score of $0-1$ at 1 year $(21 \%$ with $\mathrm{mRS}$ score of 0 and $28 \%$ with $\mathrm{mRS}$ score of 1$).{ }^{15}$ In comparison, we found $63 \%$ of our patients achieved an mRS score of $0-1$ within 1 year. The multi- 
center CONSCIOUS trials reported functional outcomes using a dichotomized version of the Glasgow Outcome Scale-Extended at 12 weeks, making a comparison with our results difficult. ${ }^{12,13}$ All-inclusive, observational singlecenter studies may present cohorts more comparable to ours. However, these studies most frequently lack detail on best functional outcome categories or have more limited follow-up. $.16,17,24,25$

The variables in our final prediction model include 2 nonmodifiable and 2 modifiable factors. The prognostic influence of early clinical grade is well known. ${ }^{7}$ As we have previously described, the prognostic accuracy is refined when using the clinical grade after neurological resuscitation. ${ }^{4}$ Although a good clinical grade was definitely the strongest factor associated with greater chances of excellent outcome in our cohort, we also found that nearly onethird of patients who presented with poor clinical grade were able to reach an excellent recovery. These numbers are higher than those reported in a previous study, which found that $16 \%$ of poor-grade patients who underwent aneurysm treatment had an MRS score of $0-1$ at 1 year..14 The negative impact of ICH on prognosis is not surprising because any motor or cognitive deficits caused by brain damage from the hematoma would impede recovery with no more than minimal symptoms. The associations of radiological infarctions and transfusion with worse outcomes have been previously reported by our group and other investigators. ${ }^{3,10,20,24}$ Nonetheless, these results highlight the need to treat cerebral ischemia aggressively and to favor a conservative transfusion strategy to maximize the chances of attaining an excellent outcome, although the exact cutoff for considering transfusion needs to be better studied.

Our study has several limitations. It is based on a single-center cohort from a tertiary referral center, which may limit the external validity of the results. We did not have 1-year outcomes in all patients. Actually, this limitation may have actually caused us to underestimate the degree of recovery in some patients because the trajectory of functional recovery over the 1st year after discharge is known to be favorable. ${ }^{17}$ A more important deficiency of our analysis is that we did not have information on quality of life or cognitive function from neuropsychometric testing. Quality of life may be reduced, and mood disorders and cognitive dysfunction can be present in a substantial proportion of patients with favorable functional scores after aSAH. ${ }^{1,22}$ However, an mRS score of $0-1$ is a close approximation as it includes only patients who believed they were back to their previous activities (before the SAH). We did not have firm standardized criteria for transfusion during the study period. As with all observational studies, the associations identified in our analysis cannot be proven to be causal.

\section{Conclusions}

A high proportion of patients can recover full function and have minimal or no long-term symptoms after aSAH. Especially for patients with good clinical grades, the therapeutic goal must be full or nearly full recovery. Preventing and aggressively treating delayed ischemia to prevent infarctions and keeping a conservative transfusion strategy may help attain this goal.

\section{References}

1. Al-Khindi T, Macdonald RL, Schweizer TA: Cognitive and functional outcome after aneurysmal subarachnoid hemorrhage. Stroke 41:e519-e536, 2010

2. Brinjikji W, Lanzino G, Rabinstein AA, Kallmes DF, Cloft HJ: Age-related trends in the treatment and outcomes of ruptured cerebral aneurysms: a study of the nationwide inpatient sample 2001-2009. AJNR Am J Neuroradiol 34:1022-1027, 2013

3. Festic E, Rabinstein AA, Freeman WD, Mauricio EA, Robinson MT, Mandrekar J, et al: Blood transfusion is an important predictor of hospital mortality among patients with aneurysmal subarachnoid hemorrhage. Neurocrit Care 18:209-215, 2013

4. Giraldo EA, Mandrekar JN, Rubin MN, Dupont SA, Zhang Y, Lanzino G, et al: Timing of clinical grade assessment and poor outcome in patients with aneurysmal subarachnoid hemorrhage. J Neurosurg 117:15-19, 2012

5. Hackett ML, Anderson CS: Health outcomes 1 year after subarachnoid hemorrhage: an international population-based study. Neurology 55:658-662, 2000

6. Helbok R, Kurtz P, Vibbert M, Schmidt MJ, Fernandez L, Lantigua $\mathrm{H}$, et al: Early neurological deterioration after subarachnoid haemorrhage: risk factors and impact on outcome. J Neurol Neurosurg Psychiatry 84:266-270, 2013

7. Jaja BN, Cusimano MD, Etminan N, Hanggi D, Hasan D, Ilodigwe D, et al: Clinical prediction models for aneurysmal subarachnoid hemorrhage: a systematic review. Neurocrit Care 18:143-153, 2013

8. Johnston SC, Selvin S, Gress DR: The burden, trends, and demographics of mortality from subarachnoid hemorrhage. Neurology 50:1413-1418, 1998

9. Kassell NF, Adams HP Jr, Torner JC, Sahs AL: Influence of timing of admission after aneurysmal subarachnoid hemorrhage on overall outcome. Report of the Cooperative Aneurysm Study. Stroke 12:620-623, 1981

10. Kramer AH, Gurka MJ, Nathan B, Dumont AS, Kassell NF, Bleck TP: Complications associated with anemia and blood transfusion in patients with aneurysmal subarachnoid hemorrhage. Crit Care Med 36:2070-2075, 2008

11. Lovelock CE, Rinkel GJ, Rothwell PM: Time trends in outcome of subarachnoid hemorrhage: population-based study and systematic review. Neurology 74:1494-1501, 2010

12. Macdonald RL, Higashida RT, Keller E, Mayer SA, Molyneux A, Raabe A, et al: Clazosentan, an endothelin receptor antagonist, in patients with aneurysmal subarachnoid haemorrhage undergoing surgical clipping: a randomised, doubleblind, placebo-controlled phase 3 trial (CONSCIOUS-2). Lancet Neurol 10:618-625, 2011

13. Macdonald RL, Higashida RT, Keller E, Mayer SA, Molyneux A, Raabe A, et al: Randomized trial of clazosentan in patients with aneurysmal subarachnoid hemorrhage undergoing endovascular coiling. Stroke 43:1463-1469, 2012

14. Mocco J, Ransom ER, Komotar RJ, Schmidt JM, Sciacca RR, Mayer SA, et al: Preoperative prediction of long-term outcome in poor-grade aneurysmal subarachnoid hemorrhage. Neurosurgery 59:529-538, 2006

15. Molyneux AJ, Kerr RS, Yu LM, Clarke M, Sneade M, Yarnold JA, et al: International Subarachnoid Aneurysm Trial (ISAT) of neurosurgical clipping versus endovascular coiling in 2143 patients with ruptured intracranial aneurysms: a randomised comparison of effects on survival, dependency, seizures, rebleeding, subgroups, and aneurysm occlusion. Lancet 366:809-817, 2005

16. Naval NS, Chang T, Caserta F, Kowalski RG, Carhuapoma 
JR, Tamargo RJ: Improved aneurysmal subarachnoid hemorrhage outcomes: a comparison of 2 decades at an academic center. J Crit Care 28:182-188, 2013

17. Navi BB, Kamel H, Hemphill JC III, Smith WS: Trajectory of functional recovery after hospital discharge for subarachnoid hemorrhage. Neurocrit Care 17:343-347, 2012

18. Nieuwkamp DJ, Setz LE, Algra A, Linn FH, de Rooij NK, Rinkel GJ: Changes in case fatality of aneurysmal subarachnoid haemorrhage over time, according to age, sex, and region: a meta-analysis. Lancet Neurol 8:635-642, 2009

19. Qureshi AI, Vazquez G, Tariq N, Suri MF, Lakshminarayan K, Lanzino G: Impact of International Subarachnoid Aneurysm Trial results on treatment of ruptured intracranial aneurysms in the United States. Clinical article. J Neurosurg 114:834-841, 2011

20. Rabinstein AA, Friedman JA, Weigand SD, McClelland RL, Fulgham JR, Manno EM, et al: Predictors of cerebral infarction in aneurysmal subarachnoid hemorrhage. Stroke 35:1862-1866, 2004

21. Rabinstein AA, Lanzino G, Wijdicks EF: Multidisciplinary management and emerging therapeutic strategies in aneurysmal subarachnoid haemorrhage. Lancet Neurol 9:504-519, 2010

22. Rinkel GJ, Algra A: Long-term outcomes of patients with aneurysmal subarachnoid haemorrhage. Lancet Neurol 10:349-356, 2011

23. Samuels O, Webb A, Culler S, Martin K, Barrow D: Impact of a dedicated neurocritical care team in treating patients with aneurysmal subarachnoid hemorrhage. Neurocrit Care 14:334-340, 2011

24. Schmidt JM, Wartenberg KE, Fernandez A, Claassen J, Rincon F, Ostapkovich ND, et al: Frequency and clinical impact of asymptomatic cerebral infarction due to vasospasm after subarachnoid hemorrhage. J Neurosurg 109:10521059,2008

25. Wartenberg KE, Schmidt JM, Claassen J, Temes RE, Frontera JA, Ostapkovich N, et al: Impact of medical complications on outcome after subarachnoid hemorrhage. Crit Care Med 34:617-623, 2006

\section{Author Contributions}

Conception and design: Lanzino, Rabinstein. Acquisition of data: Pegoli, Rabinstein. Analysis and interpretation of data: all authors. Drafting the article: Pegoli, Rabinstein. Critically revising the article: Lanzino, Mandrekar, Rabinstein. Reviewed submitted version of manuscript: all authors. Approved the final version of the manuscript on behalf of all authors: Lanzino. Statistical analysis: Mandrekar. Study supervision: Lanzino, Rabinstein.

\section{Correspondence}

Giuseppe Lanzino, Department of Neurosurgery, Mayo Clinic, 200 First St. SW, Rochester, MN 55905. email: lanzino. giuseppe@mayo.edu. 